\title{
ROUGH INTEGERS WITH A DIVISOR IN A GIVEN INTERVAL
}

\author{
KEVIN FORD
}

\begin{abstract}
We determine, up to multiplicative constants, the number of integers $n \leqslant x$ that have no prime factor $\leqslant w$ and a divisor in $(y, 2 y]$. Our estimate is uniform in $x, y, w$. We apply this to determine the order of the number of distinct integers in the $N \times N$ multiplication table which are free of prime factors $\leqslant w$, and the number of distinct fractions of the form $\frac{a_{1} a_{2}}{b_{1} b_{2}}$ with $1 \leqslant a_{1} \leqslant b_{1} \leqslant N$ and $1 \leqslant a_{2} \leqslant b_{2} \leqslant N$.
\end{abstract}

\section{INTRODUCTION}

In the paper [5], the author established the order of growth of $H(x, y, z)$, the number of integers $n \leqslant x$ which have a divisor in the interval $(y, z]$, for all $x, y, z$. An important special case is

$$
H(x, y, 2 y) \asymp \frac{x}{(\log y)^{\mathcal{E}}\left(\log _{2} y\right)^{3 / 2}} \quad(3 \leqslant y \leqslant \sqrt{x}),
$$

where

$$
\mathcal{E}=1-\frac{1+\log _{2} 2}{\log 2}=0.086071332 \ldots
$$

A shorter, more direct proof of the order of magnitude bounds in the special case (1.1) is given in [6]. More on the history of estimations of $H(x, y, z)$, further applications and references may be found in $[\mathbf{5}$.

A number of recent aplications have required similar bounds, but where the underlying set of integers $n$ is restricted to a special set, e.g. the set of shifted primes ([5, Theorem 6,7], [9]) or the values of a polynomial [2, 1, 12, 13, 7]. More generally, we define

$$
H(x, y, z ; \mathcal{A})=\mid\{n \leqslant x, n \in \mathcal{A}: d \mid n \text { for some } d \in(y, z]\} \mid .
$$

Another natural set to consider is $\mathcal{R}_{w}$, the set of integers with no prime factor $p \leqslant w$; called $w$-rough numbers by some authors. Here we determinte the exact order of growth of $H\left(x, y, 2 y ; \mathcal{R}_{w}\right)$ for all $x, y, w$; the more general quantity $H\left(x, y, z ; \mathcal{R}_{w}\right)$ can be estimated by similar methods, although there are many cases depending on the relative size of the parameters $w, x, y, z$.

Theorem 1. Suppose that $4 \leqslant y \leqslant \sqrt{x}, 4 \leqslant w \leqslant y / 8$ and writ 1$]=\frac{\log _{2} w}{\log _{2} y}$.

(i) When $1-1 / \log 4 \leqslant \delta \leqslant 1$ we have

$$
H\left(x, y, 2 y ; \mathcal{R}_{w}\right)-H\left(x / 2, y, 2 y ; \mathcal{R}_{w}\right) \gg \frac{x}{\log ^{2} w} \gg H\left(x, y, 2 y ; \mathcal{R}_{w}\right) .
$$

Date: September 19, 2019.

2000 Mathematics Subject Classification: Primary 11N25; Secondary 62G30.

Research supported by National Science Foundation grant DMS-1802139.

${ }^{1}$ The notation $\log _{2} x$ stands for $\log \log x$. 
(ii) When $0 \leqslant \delta<1-1 / \log 4$, we have

$$
\begin{gathered}
H\left(x, y, 2 y ; \mathcal{R}_{w}\right)-H\left(x / 2, y, 2 y ; \mathcal{R}_{w}\right) \gg x \delta B(w, y)(\log y)^{-\mathcal{E}+\frac{\log (1-\delta)}{\log 2}} \gg H\left(x, y, 2 y ; \mathcal{R}_{w}\right), \\
\text { where } \quad B(w, y)=\min \left(1,\left(\log _{2} y\right)^{-1 / 2}((1-\delta) \log 4-1)^{-1}\right) .
\end{gathered}
$$

Remark 1. Some special cases are worth noting. From Theorem 1 we have

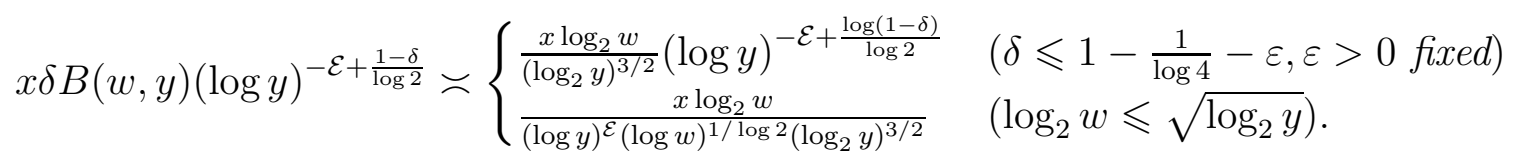

Remark 2. When $y>\sqrt{x}$, one can obtain similar results by using the duality $d \mid n \Longleftrightarrow$ $(n / d) \mid n$. That is, if $x / 2<n \leqslant x$, then $d \mid n$ with $y<d \leqslant 2 y$ is equivalent to $d^{\prime} \mid n$ with $d^{\prime} \asymp x / y$.

We illustrate the utility of Theorem 1 with two applications. The first is related to the well-know multiplication table problem of Erdös [3, 4], which asks for estimates on the number, $M(N)$, of distinct integers in an $N \times N$ multiplication table. In [5] the author proved, using (1.1), that

$$
M(N) \asymp \frac{N^{2}}{(\log N)^{\mathcal{E}}\left(\log _{2} N\right)^{3 / 2}} .
$$

More generally, consider the restricted multiplication table problem of bounding $M(N ; \mathcal{A})$, the number of distinct entries in an $N \times N$ multiplication table that belong to the set $\mathcal{A}$. For example, when $\lambda \neq 0$ is fixed and $\mathcal{A}=\{p+\lambda: p$ prime $\}$, the order of $M(N ; \mathcal{A})$ was determined in [5, Theorem 6] (upper bound) and [9] (lower bound).

Observe that $M\left(N ; \mathcal{R}_{w}\right)=1$ when $w \geqslant N$.

Corollary 2. Uniformly for $4 \leqslant w \leqslant N / 2$, we have

$$
M\left(N ; \mathcal{R}_{w}\right) \asymp \begin{cases}\frac{N^{2}}{\log ^{2} w} & \text { if } \log w \geqslant(\log N)^{1-1 / \log 4} \\ N^{2} \delta B(w, N)(\log N)^{-\mathcal{E}+\frac{\log (1-\delta)}{\log 2}} & \text { if } \log w=(\log y)^{\delta}, \delta \leqslant 1-\frac{1}{\log 4} .\end{cases}
$$

Proof. If $\sqrt{N}<w \leqslant N / 2$, then $M\left(N ; \mathcal{R}_{w}\right)$ counts entries in the multiplication table which are primes in $(w, N]$ or the product of two such primes. The desired bounds follow. If $4 \leqslant w \leqslant \sqrt{N}$, we use the inequalities

$$
H\left(\frac{N^{2}}{4}, \frac{N}{4}, \frac{N}{2} ; \mathcal{R}_{w}\right) \leqslant M\left(N ; \mathcal{R}_{w}\right) \leqslant \sum_{k \geqslant 0} H\left(\frac{N^{2}}{2^{k}}, \frac{N}{2^{k+1}}, \frac{N}{2^{k}} ; \mathcal{R}_{w}\right) .
$$

The proof is easy: consider $a b \in \mathcal{R}_{w}, a \leqslant N$ and $b \leqslant N$. If $\frac{N}{4}<a \leqslant \frac{N}{2}$ and $a b \leqslant \frac{N^{2}}{4}$, then $b \leqslant N$ and this proves the lower bound. The upper bound comes from taking $\frac{N}{2^{k+1}}<a \leqslant \frac{N}{2^{k}}$ for some non-negative integer $k$. The desired bound for $M\left(N ; \mathcal{R}_{w}\right)$ now follow from Theorem 1, since we have $H\left(x, y, 2 y ; \mathcal{R}_{w}\right) \asymp x f(y, w)$ where $f(u, w) \asymp f(y, w)$ for $\log u \asymp \log y$.

Next, we consider the "Farey fraction multiplication table". Let $\mathcal{F}_{N}$ of Farey fractions of order $N$, i.e.,

$$
\mathcal{F}_{N}=\left\{\frac{a}{b}: 1 \leqslant a \leqslant b \leqslant N,(a, b)=1\right\}
$$


In private conversation, Igor Shparlinski asked the author about the size of the product set $\mathcal{F}_{N} \mathcal{F}_{N}$ (in general, for sets $\mathcal{A}, \mathcal{B} \in \mathbb{Z}, \mathcal{A B}$ denotes the product set $\{a b: a \in \mathcal{A}, b \in \mathcal{B}\}$ ).

Corollary 3. We have

$$
M(N)^{2} \ll\left|\mathcal{F}_{N} \mathcal{F}_{N}\right| \leqslant M(N)^{2} .
$$

Consequently, by (1.2), we have

$$
\left|\mathcal{F}_{N} \mathcal{F}_{N}\right| \asymp \frac{N^{2}}{(\log N)^{\mathcal{E}}\left(\log _{2} N\right)^{3 / 2}} .
$$

Proof. The upper bound is trivial, and thus the real work is on the lower bound. We achieve this by placing restrictions on the fractions, firstly by putting them in dyadic intervals and secondly by removing those elements divisible by small primes. To this end, define

$$
\mathcal{A}_{N}=\{n: N / 2 \leqslant n \leqslant N\}, \quad \mathcal{A}_{N}^{(w)}=\mathcal{A}_{N} \cap \mathcal{R}_{w} .
$$

Let $w$ be a large, fixed constant. A simple inclusion-exclusion argument yields (here $p$ denotes a prime in the sums)

$$
\begin{aligned}
\left|\mathcal{F}_{N} \mathcal{F}_{N}\right| & \geqslant\left|\left\{\frac{a_{1} a_{2}}{b_{1} b_{2}}: a_{1}, a_{2} \in \mathcal{A}_{N / 2}^{(w)} ; b_{1}, b_{2} \in \mathcal{A}_{N}^{(w)} ;\left(a_{1} a_{2}, b_{1} b_{2}\right)=1\right\}\right| \\
& \geqslant\left|\mathcal{A}_{N / 2}^{(w)} \mathcal{A}_{N / 2}^{(w)}\right| \cdot\left|\mathcal{A}_{N}^{(w)} \mathcal{A}_{N}^{(w)}\right|-\sum_{w<p \leqslant N / 2}\left|\mathcal{A}_{N / 2}^{(w)} \mathcal{A}_{N / 2 p}^{(w)}\right| \cdot\left|\mathcal{A}_{N}^{(w)} \mathcal{A}_{N / p}^{(w)}\right| \\
& \geqslant\left|\mathcal{A}_{N / 2}^{(w)} \mathcal{A}_{N / 2}^{(w)}\right| \cdot\left|\mathcal{A}_{N}^{(w)} \mathcal{A}_{N}^{(w)}\right|-\sum_{w<p \leqslant N / 2}\left|\mathcal{A}_{N / 2} \mathcal{A}_{N / 2 p}\right| \cdot\left|\mathcal{A}_{N} \mathcal{A}_{N / p}\right|
\end{aligned}
$$

It is clear that for $M \leqslant N$ we have

$$
\left|\mathcal{A}_{N} \mathcal{A}_{M}\right| \leqslant H(M N, M / 2, M)
$$

and we deduce from (1.1) that

$$
\sum_{w<p \leqslant N / 2}\left|\mathcal{A}_{N / 2} \mathcal{A}_{N / 2 p}\right| \cdot\left|\mathcal{A}_{N} \mathcal{A}_{N / p}\right| \ll \sum_{p>w} \frac{N^{4}}{p^{2}(\log (N / p))^{2 \mathcal{E}}\left(\log _{2}(N / p)\right)^{3}} \ll \frac{M(N)^{2}}{w \log w} .
$$

We also have the lower bound

$$
\left|\mathcal{A}_{N}^{(w)} \mathcal{A}_{M}^{(w)}\right| \geqslant H\left(M N, M / 2, M ; \mathcal{R}_{w}\right)-H\left(M N / 2, M / 2, M ; \mathcal{R}_{w}\right) .
$$

It follows that

$$
\begin{aligned}
\left|\mathcal{F}_{N} \mathcal{F}_{N}\right| \geqslant\left(H\left(\frac{N^{2}}{4}, \frac{N}{4}, \frac{N}{2} ; \mathcal{R}_{w}\right)-H\left(\frac{N^{2}}{8}, \frac{N}{4}, \frac{N}{2} ; \mathcal{R}_{w}\right)\right)( & \left.H\left(N^{2}, \frac{N}{2}, N ; \mathcal{R}_{w}\right)-H\left(\frac{N^{2}}{2}, \frac{N}{2}, N ; \mathcal{R}_{w}\right)\right)- \\
& -O\left(\frac{N^{4}}{(\log N)^{2 \mathcal{E}}\left(\log _{2} N\right)^{3}(w \log w)}\right) .
\end{aligned}
$$

Inserting Theorem 1 into the estimate (1.3), and taking $w$ to be a sufficiently large constant, we obtain the lower bound in Corollary 3 . 
1.1. Notation. Let $\tau(n)$ be the number of positive divisors of $n$, and $\tau(n ; y, z)$ denotes the number of divisors of $n$ within the interval $(y, z]$. Let $\omega(n)$ be the number of distinct prime divisors of $n$. Let $P^{+}(n)$ be the largest prime factor of $n$ and let $P^{-}(n)$ be the smallest prime factor of $n$. Adopt the notational conventions $P^{+}(1)=0$ and $P^{-}(1)=\infty$. Constants implied by $O$, « and $\asymp$ are absolute. The notation $f \asymp g$ means $f \ll g$ and $g \ll f$. The symbol $p$ will always denote a prime. Lastly, $\log _{2} x$ denotes $\log \log x$.

1.2. Heuristics. Here we give a short heuristic argument to justify the formulas in Theorem 1. This is similar to the heuristics givin in [5, 6].

Write $n=n^{\prime} n^{\prime \prime}$, where $n^{\prime}$ is composed only of primes in $(w, 2 y]$ and $n^{\prime \prime}$ is composed only of primes $>2 y$. For simplicity, assume $n^{\prime}$ is squarefree and $n^{\prime} \leqslant y^{100}$. Assume for the moment that the set $D\left(n^{\prime}\right)=\left\{\log d: d \mid n^{\prime}\right\}$ is approximately uniformly distributed in $\left[0, \log n^{\prime}\right]$. If $n^{\prime}$ has $k$ prime factors, then $\tau\left(n^{\prime}\right)=2^{k}$ and we thus expect that $\tau\left(n^{\prime}, y, 2 y\right) \geqslant 1$ with probability about

$$
\min \left(1, \frac{2^{k}}{\log y}\right)
$$

This expression changes behavior at $k=k_{0}:=\left\lfloor\frac{\log _{2} y}{\log 2}\right\rfloor$. The number of $n \leqslant x$ with $n^{\prime} \in \mathcal{R}_{w}$ and $\omega\left(n^{\prime}\right)=k$ is of size

$$
\frac{x}{\log y} \frac{\left(\log _{2} y-\log _{2} w\right)^{k}}{k !}
$$

and we obtain a heuristic estimate for $H\left(x, y, 2 y ; \mathcal{R}_{w}\right)$ of order

$$
\frac{x}{\log ^{2} y}\left[\sum_{k \leqslant k_{0}} \frac{\left(2 \log _{2} y-2 \log _{2} w\right)^{k}}{k !}+(\log y) \sum_{k \geqslant k_{0}} \frac{\left(\log _{2} y-\log _{2} w\right)^{k}}{k !}\right] .
$$

The first sum always dominates, since the second sum is dominated by the first summand ( $k_{0}$ is always much larger than $\log _{2} y-\log _{2} w$ ). The behavior of the first sum over $k$ depends on the relative sizes of $k_{0}$ and $2 \log _{2} y-2 \log _{2} w$. If $k_{0}>2 \log _{2} y-2 \log _{2} w$, that is, $\log w \geqslant$ $(\log y)^{1-1 / \log 4}$, the first contains the "peak" and we obtain

$$
H\left(x, y, 2 y ; \mathcal{R}_{w}\right) \approx \frac{x}{\log ^{2} y} e^{2 \log _{2} y-2 \log _{2} w}=\frac{x}{\log ^{2} w} .
$$

For smaller $w$, we are summing the left tail of the Poisson distribution and standard bounds (see e.g. Lemma 2.4 below) yield

$$
H\left(x, y, 2 y ; \mathcal{R}_{w}\right) \approx x B(y, w)(\log y)^{-\mathcal{E}+\frac{\log (1-\delta)}{\log 2}} .
$$

This latter expression is too large by a factor $1 / \delta$, and this stems from the uniformity assumption about $D\left(n^{\prime}\right)$, which turns out to be false for all but a proportion $\delta$ of these integers. Fluctuations in the distribution of the prime factors of $n^{\prime}$ lead to clustering of the divisors; more details can be found in [5, 6]. As in [5, 6], we really should be considering those $n^{\prime}$ which have nicely distributed divisors, and a useful measure of how nicely distributed the divisors are is the function

$$
L(a)=\operatorname{meas} \mathscr{L}(a), \quad \mathscr{L}(a)=\bigcup_{d \mid a}[-\log 2+\log d, \log d) .
$$


Adjusting our heuristic, we see that the probability that $\tau\left(n^{\prime}, y, 2 y\right) \geqslant 1$ should be about $L\left(n^{\prime}\right) / \log y$, which is $\gg 1 / \log y$ on a set of $n^{\prime}$ of density $\delta$.

\section{Preliminaries}

Lemma 2.1 ([6, Lemma 3.1]). We have

(i) $L(a) \leqslant \min (\tau(a) \log 2, \log 2+\log a)$;

(ii) If $(a, b)=1$, then $L(a b) \leqslant \tau(b) L(a)$;

(iii) If $p_{1}<\cdots<p_{k}$, then

$$
L\left(p_{1} \cdots p_{k}\right) \leqslant \min _{0 \leqslant j \leqslant k} 2^{k-j}\left(\log \left(p_{1} \cdots p_{j}\right)+\log 2\right) .
$$

Let $\mathscr{P}(a, b)$ be the set of all squarefree positive integers composed only of primes in $(a, b]$. We adopt the convention that $1 \in \mathscr{P}(a, b)$ for any $a, b$.

Lemma 2.2. (a) For $t \geqslant w \geqslant 2$ and $k \geqslant 0$ we have

$$
\sum_{\substack{a \in \mathscr{P}(w, t) \\ \omega(a)=k}} \frac{1}{a} \leqslant \frac{\left(\log _{2} t-\log _{2} w+O(1)\right)^{k}}{k !} .
$$

(b) For $t \geqslant w \geqslant 2$ and $k \geqslant 1$ we have

$$
\sum_{\substack{a \in \mathscr{P}(w, t) \\ \omega(a)=k}} \frac{\log a}{a} \ll(1+\log (t / w)) \frac{\left(\log _{2} t-\log _{2} w+O(1)\right)^{k-1}}{(k-1) !} .
$$

(c) For $2 \leqslant w \leqslant s \leqslant t$, we have

$$
\sum_{a \in \mathscr{P}(w, t)} \frac{L(a)}{a} \ll\left(\frac{\log t}{\log s}\right)^{2} \sum_{a \in \mathscr{P}(w, s)} \frac{L(a)}{a} .
$$

Proof. Item (a) is immediate from

$$
\sum_{\substack{a \in \mathscr{P}(w, t) \\ \omega(a)=k}} \frac{1}{a} \leqslant \frac{1}{k !}\left(\sum_{w<p \leqslant t} \frac{1}{p}\right)^{k}
$$

and Mertens' estimate. For item (b), we have

$$
\sum_{\substack{a \in \mathscr{P}(w, t) \\ \omega(a)=k}} \frac{\log a}{a}=\sum_{\substack{a \in \mathscr{P}(w, t) \\ \omega(a)=k}} \frac{1}{a} \sum_{p \mid a} \log p \leqslant \sum_{w<p \leqslant t} \frac{\log p}{p} \sum_{\substack{a \in \mathscr{P}(w, t) \\ \omega(a)=k-1}} \frac{1}{a} .
$$

The desired inequality follows from part (a) and Mertens' estimates. For part (c), we factor each $a \in \mathscr{P}(w, t)$ uniquely as $a=a_{1} a_{2}$ with $a_{1} \in \mathscr{P}(w, s)$ and $a_{2} \in \mathscr{P}(s, t)$. Then, using 
Lemma 2.1 (ii) we deduce that

$$
\begin{aligned}
\sum_{a \in \mathscr{P}(w, t)} \frac{L(a)}{a} & \leqslant \sum_{a_{1} \in \mathscr{P}(w, s)} \frac{L\left(a_{1}\right)}{a_{1}} \sum_{a_{2} \in \mathscr{P}(s, t)} \frac{\tau\left(a_{2}\right)}{a_{2}} \\
& =\prod_{s<p \leqslant t}\left(1+\frac{2}{p}\right) \sum_{a_{1} \in \mathscr{P}(w, s)} \frac{L\left(a_{1}\right)}{a_{1}} .
\end{aligned}
$$

The desired inequality follows from Mertens' estimates.

The following is a standard sieve bound, see e.g. [8].

Lemma 2.3. (a) Uniformly for $x \geqslant 2 z \geqslant 4$, we have

$$
\left|\left\{x / 2<n \leqslant x: P^{-}(n)>z\right\}\right| \gg \frac{x}{\log z} .
$$

Uniformly for $x \geqslant z \geqslant 2$ we have

$$
\left|\left\{n \leqslant x: P^{-}(n)>z\right\}\right| \ll \frac{x}{\log z} .
$$

Finally, we quote standard bounds on the Poisson distribution, see e.g. the results in Section 4 of [1].

Lemma 2.4. Uniformly for $h \leqslant m \leqslant x$, we have

$$
\sum_{h \leqslant k \leqslant m} \frac{x^{k}}{k !} \asymp \min \left(\sqrt{x}, \frac{x}{x-m}, m-h+1\right) \frac{x^{m}}{m !} .
$$

\section{LOCAL-TO-GLOBAL ESTIMATES}

Following a kind of local-to-global principle first utilized in [5], we bound $H\left(x, y, 2 y ; \mathcal{R}_{w}\right)$ in terms of the function $L(a)$. This justifies the heuristic presented in Section 1.2.

Lemma 3.1. If $w \leqslant y^{1 / 15}$ and $y \leqslant \sqrt{x}$, then

$$
H\left(x, y, 2 y ; \mathcal{R}_{w}\right)-H\left(x / 2, y, 2 y ; \mathcal{R}_{w}\right) \gg \frac{x}{\log ^{2} y} \sum_{a \in \mathscr{P}(w, y)} \frac{L(a)}{a} .
$$

If $w \leqslant y \leqslant \sqrt{x}$ and $w \leqslant y^{1 / 10}$, then

$$
H\left(x, y, 2 y ; \mathcal{R}_{w}\right) \ll \frac{x}{\log ^{2} y} \sum_{a \in \mathscr{P}(w, y)} \frac{L(a)}{a} .
$$

Proof. We begin with the lower bound. We may assume without loss of generality that $y \geqslant y_{0}$, where $y_{0}$ is a sufficiently large constant, because in the case $y<y_{0}$, for any prime $p \in(y, 2 y]$ (such $p$ exists by Bertrand's Postulate) and we see that

$$
H\left(x, y, 2 y ; \mathcal{R}_{w}\right)-H\left(x / 2, y, 2 y ; \mathcal{R}_{w}\right) \gg x / p \gg_{y_{0}} x
$$

Consider integers $n=a p_{1} p_{2} b \in(x / 2, x]$ with $P^{-}(a)>w, p_{1}$ and $p_{2}$ prime, satisfying the inequalities

$$
a \leqslant y^{1 / 5}<p_{1}<p_{2} \leqslant \frac{1}{4} y^{4 / 5}<P^{-}(b),
$$


and with $\log \left(y / p_{1} p_{2}\right) \in \mathscr{L}(a)$. The last condition implies that $\tau\left(a p_{1} p_{2}, y, 2 y\right) \geqslant 1$, and we also have that $P^{-}(n)>w$. Since $y^{4 / 5} \leqslant y / a<p_{1} p_{2} \leqslant 2 y$, we have $x / a p_{1} p_{2} \geqslant x /\left(2 y^{6 / 5}\right) \geqslant \frac{1}{2} y^{4 / 5}$. Thus, by Lemma 2.3, for each triple $\left(a, p_{1}, p_{2}\right)$, the number of possible $b$ is $\gg \frac{x}{a p_{1} p_{2} \log y}$. Now $\mathscr{L}(a)$ is the disjoint union of intervals of length $\geqslant \log 2$ contained in $[-\log 2, \log a]$. For each such interval $[u, v)$, Mertens' estimate implies that

$$
\sum_{\substack{u \leqslant \log \left(y / p_{1} p_{2}\right)<v \\ y^{1 / 5}<p_{1}<p_{2}<\frac{1}{4} y^{4 / 5}}} \frac{1}{p_{1} p_{2}} \geqslant \sum_{8 y^{1 / 5}<p_{1}<y^{2 / 5}} \frac{1}{p_{1}} \sum_{y e^{-v} / p_{1}<p_{2} \leqslant y e^{-u} / p_{1}} \frac{1}{p_{2}} \gg \frac{v-u}{\log y} .
$$

Here we made use of the estimate $v \leqslant \log a \leqslant \frac{1}{5} \log y$ which implies that $y e^{-v} / p_{1} \geqslant y^{2 / 5}>p_{1}$. Thus, with $a$ fixed, the sum of $\frac{1}{p_{1} p_{2}}$ is $\gg \frac{L(a)}{\log y}$ and we obtain

$$
H\left(x, y, 2 y ; \mathcal{R}_{w}\right)-H\left(x / 2, y, 2 y ; \mathcal{R}_{w}\right) \gg \frac{x}{\log ^{2} y} \sum_{\substack{a \leqslant y^{1 / 5} \\ P^{-}(a)>w}} \frac{L(a)}{a} .
$$

We to replace the sum over $a$ with an unbounded set which is muliplicatively more convenient, starting with

$$
\sum_{\substack{a \leqslant y^{1 / 5} \\ P^{-}(a)>w}} \frac{L(a)}{a} \geqslant \sum_{\substack{a \leqslant y^{1 / 5} \\ a \in \mathscr{P}\left(w, y^{1 / 15}\right)}} \frac{L(a)}{a} \geqslant \sum_{a \in \mathscr{P}\left(w, y^{1 / 15}\right)} \frac{L(a)}{a}\left(1-\frac{\log a}{\log \left(y^{1 / 5}\right)}\right) .
$$

Break this into two sums, the first being what we want and the second involving

$$
\sum_{a \in \mathscr{P}\left(w, y^{1 / 15}\right)} \frac{L(a) \log a}{a}=\sum_{a \in \mathscr{P}\left(w, y^{1 / 15}\right)} \frac{L(a)}{a} \sum_{p \mid a} \log p=\sum_{w<p \leqslant y^{1 / 15}} \frac{\log p}{p} \sum_{\substack{b \in \mathscr{P}\left(w, y^{1 / 15}\right) \\ p \nmid b}} \frac{L(p b)}{b} .
$$

Using the trivial relation $L(p b) \leqslant 2 L(b)$ which comes from Lemma 2.1 (ii), and Mertens' estimate, we have

$$
\sum_{\substack{a \leqslant y^{1 / 5} \\ P^{-}(a)>w}} \frac{L(a)}{a} \geqslant \sum_{a \in \mathscr{P}\left(w, y^{1 / 15}\right)} \frac{L(a)}{a}\left(1-\frac{2 \log \left(y^{1 / 15}\right)+O(1)}{\log \left(y^{1 / 5}\right)}\right) \gg \sum_{a \in \mathscr{P}\left(w, y^{1 / 15}\right)} \frac{L(a)}{a} .
$$

An application of Lemma 2.2 (c) concludes the proof of the lower bound.

For the upper bound, we first relate $H\left(x, y, 2 y ; \mathcal{R}_{w}\right)$ to $H^{*}\left(x, y, 2 y ; \mathcal{R}_{w}\right)$, the number of squarefree integers $n \leqslant x$ with $P^{-}(n)>w$ and $\tau(n, y, z) \geqslant 1$. Write $n=n^{\prime} n^{\prime \prime}$, where $n^{\prime}$ is squarefree, $n^{\prime \prime}$ is squarefull and $\left(n^{\prime}, n^{\prime \prime}\right)=1$. The number of $n \leqslant x$ with $n^{\prime \prime}>\log ^{10} y$ is

$$
\leqslant x \sum_{n^{\prime \prime}>\log ^{10} y} \frac{1}{n^{\prime \prime}} \ll \frac{x}{\log ^{5} y} .
$$

If $n^{\prime \prime} \leqslant \log ^{10} y$, then for some $f \mid n^{\prime \prime}, n^{\prime}$ has a divisor in $(y / f, 2 y / f]$, hence

$$
H\left(x, y, 2 y ; \mathcal{R}_{w}\right) \leqslant \sum_{\substack{n^{\prime \prime} \leqslant \log ^{10} y \\ P^{-}(n)>w}} \sum_{f \mid n^{\prime \prime}} H^{*}\left(\frac{x}{n^{\prime \prime}}, \frac{y}{f}, \frac{2 y}{f} ; \mathcal{R}_{w}\right)+O\left(\frac{x}{\log ^{5} y}\right) .
$$


Let $w_{0}$ be a sufficiently large absolute constant. It suffices to prove the upper bound for $w \geqslant w_{0}$, for the case $w<w_{0}$ follows from the case $w=w_{0}$. In the sum,

$$
y / f \leqslant y \leqslant\left(x / n^{\prime \prime}\right)^{1 / 2} \log ^{5} y \leqslant\left(x / n^{\prime \prime}\right)^{5 / 9}
$$

for large enough $w_{0}$. We will show that for $w_{0} \leqslant y_{1} \leqslant x_{1}^{5 / 9}$,

$$
H^{*}\left(x_{1}, y_{1}, 2 y_{1} ; \mathcal{R}_{w}\right) \ll x_{1} \max _{t \geqslant y_{1}^{3 / 4}} \frac{1}{\log ^{2} t} \sum_{a \in \mathscr{P}(w, t)} \frac{L(a)}{a} .
$$

It follows from (3.2) and (3.1) that

$$
\begin{aligned}
H\left(x, y, 2 y ; \mathcal{R}_{w}\right) & \ll \sum_{\substack{n^{\prime \prime} \leqslant \log ^{10} y \\
P^{-}(n)>w}} \frac{x}{n^{\prime \prime}} \sum_{f \mid n^{\prime \prime}} \max _{t \geqslant(y / f)^{3 / 4}} \frac{1}{\log ^{2} t} \sum_{a \in \mathscr{P}(w, t)} \frac{L(a)}{a} \\
& \ll x \max _{t \geqslant y^{2 / 3}} \frac{1}{\log ^{2} t} \sum_{a \in \mathscr{P}(w, t)} \frac{L(a)}{a} \sum_{\substack{n^{\prime \prime} \leqslant \log ^{10} y \\
P^{-}(n)>w}} \frac{\tau\left(n^{\prime \prime}\right)}{n^{\prime \prime}} .
\end{aligned}
$$

The lemma follows by noting that the inner sum over squarefull $n^{\prime \prime}$ is $O(1)$, using the relative estimate in Lemma 2.2 (c) with $s=y^{2 / 3}$, and finally noting that $\mathscr{P}\left(w, y^{2 / 3}\right) \subseteq \mathscr{P}(w, y)$.

It remains to prove (3.2). The right side is $\gg x_{1} / \log ^{2} y_{1}$ since $L(1)=\log 2$, and hence it suffices to count those $n \in\left(x_{1} / \log ^{2} y_{1}, x_{1}\right]$. We'll count separately those $n \in$ $\left(x_{1} / 2^{r+1}, x_{1} / 2^{r}\right]$ for some integer $r, 0 \leqslant r \leqslant 5 \log _{2} y_{1}$. Let $\mathscr{A}$ be the set of squarefree integers $n \in\left(x_{1} / 2^{r+1}, x_{1} / 2^{r}\right]$ with a divisor in $\left(y_{1}, 2 y_{1}\right]$. Put $z_{1}=2 y_{1}, y_{2}=\frac{x_{1}}{2^{r+2} y_{1}}, z_{2}=\frac{x_{1}}{2^{r} y_{1}}$. If $n \in \mathscr{A}$, then $n=m_{1} m_{2}$ with $y_{i}<m_{i} \leqslant z_{i}(i=1,2)$. For some $j \in\{1,2\}$ we have $p=P^{+}\left(m_{j}\right)<P^{+}\left(m_{3-j}\right)$; in particular, $p$ is not the largest prime factor of $n$. Fixing $j$, we may write $n=a b p$, where $P^{+}(a)<p<P^{-}(b)$ and $b>p$. Since $\tau\left(a p, y_{j}, z_{j}\right) \geqslant 1$, we have $y_{j} / a \leqslant p \leqslant z_{j}$. By Lemma 2.3 and the fact that $b>p$, given $a$ and $p$, the number of choices for $b$ is

$$
\ll \frac{x_{1}}{2^{r} a p \log p} \leqslant \frac{x_{1}}{2^{r} a p \log \max \left(P^{+}(a), y_{j} / a\right)},
$$

Now $a$ has a divisor in $\left(y_{j} / p, z_{j} / p\right]$, and thus $\log \left(y_{j} / p\right) \in \mathscr{L}(a)$ or $\log \left(2 y_{j} / p\right) \in \mathscr{L}(a)$. Since $\mathscr{L}(a)$ is the disjoint union of intervals of length $\geqslant \log 2$ with total measure $L(a)$, by repeated use of Mertens' estimate we obtain

$$
\sum_{\substack{\log \left(c y_{j} / p\right) \in \mathscr{L}(a) \\ p \geqslant P^{+}(a)}} \frac{1}{p} \ll \frac{L(a)}{\log \max \left(P^{+}(a), y_{j} / a\right)} \quad(c=1,2) .
$$

Since $y_{j} \geqslant y_{1}^{4 / 9} / 2^{r+2} \geqslant y_{1}^{3 / 4}$, we have that

$$
H^{*}\left(x, y, 2 y ; \mathcal{R}_{w}\right) \ll \sum_{0 \leqslant r \leqslant 5 \log _{2} y_{1}} \frac{x_{1}}{2^{r}} \sum_{t \in\left\{4 y_{1}, 4 y_{2}\right\}} \sum_{a \in \mathscr{P}(w, t)} \frac{L(a)}{a \log ^{2}\left(P^{+}(a)+t /(4 a)\right)} .
$$

We have $4 y_{j} \geqslant y_{1}^{4 / 5} / 2^{r} \geqslant y_{1}^{3 / 4}$ for any $j$ and any $r$. Also, by [10, Lemma 2.2],

$$
\sum_{a \in \mathscr{P}(w, t)} \frac{L(a)}{a \log ^{2}\left(t /(4 a)+P^{+}(a)\right)} \ll \frac{1}{\log ^{2} t} \sum_{a \in \mathscr{P}(w, t)} \frac{L(a)}{a} .
$$


Summing over $r$, we deduce (3.2).

\section{Proof of theorem 1: LOWER Bounds}

We first deal with simple cases. Let $w_{0}$ be a sufficiently large constant and $\varepsilon>0$ a sufficiently small constant. Firstly, if $y \leqslant w_{0}$, then Bertrand's postulate implies that there is a prime $p \in(y, 2 y]$ and therefore

$$
H\left(x, y, 2 y ; \mathcal{R}_{w}\right)-H_{z}\left(x / 2, y, 2 y ; \mathcal{R}_{w}\right) \geqslant \#\{x / 2<n \leqslant x: p \mid n\} \gg x .
$$

Also, if $w \leqslant w_{0}<y$ and $w \leqslant y / 8$, then

$$
H\left(x, y, 2 y ; \mathcal{R}_{w}\right)-H\left(x / 2, y, 2 y ; \mathcal{R}_{w}\right) \geqslant H\left(x, y, 2 y ; \mathcal{R}_{w_{0}}\right)-H\left(x / 2, y, 2 y ; \mathcal{R}_{w_{0}}\right)
$$

and the desired bound follows from the case $w=w_{0}$. Thirdly, when $y>w_{0}$ and $y^{\varepsilon}<w \leqslant$ $y / 8$, we consider two caess: (a) $y \leqslant \sqrt{x / 8}$ and (b) $\sqrt{x / 8}<y \leqslant \sqrt{x}$. In case (a), consider $n=p m$ where $y<p \leqslant 2 y<P^{-}(m)$. Since $x / p \geqslant 4 y$ for all such $p$, Lemma 2.3 implies

$$
\begin{aligned}
H\left(x, y, 2 y ; \mathcal{R}_{w}\right)-H\left(x / 2, y, 2 y ; \mathcal{R}_{w}\right) & \geqslant \sum_{y<p \leqslant 2 y} \#\left\{x / 2 p<n \leqslant x / p: P^{-}(n)>2 y\right\} \\
& \gg \sum_{y<p \leqslant 2 y} \frac{x}{p \log y} \gg \frac{x}{\log ^{2} w} .
\end{aligned}
$$

In the case $(\mathrm{b}) \sqrt{x / 8}<y \leqslant \sqrt{x}$, consider $n=p m$ where $y<p \leqslant 2 y$ and $P^{-}(m)>y / 8$. Such $n$ have at most three prime factors larger than $y$, hence at most three representations in this form. Since $x / p \geqslant 2 y / 8$, Lemma 2.3 similarly implies that

$$
\begin{aligned}
H\left(x, y, 2 y ; \mathcal{R}_{w}\right)-H\left(x / 2, y, 2 y ; \mathcal{R}_{w}\right) & \geqslant \frac{1}{3} \sum_{y<p \leqslant 2 y} \#\left\{x / 2 p<n \leqslant x / p: P^{-}(n)>y / 8\right\} \\
& \gg \sum_{y<p \leqslant 2 y} \frac{x}{p \log y} \gg \frac{x}{\log ^{2} w} .
\end{aligned}
$$

From now on, we assume

$$
w_{0}<w \leqslant y^{\varepsilon} .
$$

We begin with the local-to-global estimate for $H\left(x, y, 2 y ; \mathcal{R}_{w}\right)$ given in Lemma 3.1, and relate $L(a)$ to counts of pairs of divisors which are close together. Evidently,

$$
L(a) \geqslant(\log 2) \#\{d \mid a: \tau(a, d, 2 d)=0)\} \geqslant(\log 2)\left(\tau(a)-W^{*}(a)\right),
$$

where

$$
W^{*}(a)=\#\left\{d\left|a, d^{\prime}\right| a: d<d^{\prime} \leqslant 2 d\right\} .
$$

We will apply (4.2) with integers whose prime factors are localized. As in [6], partition the primes into sets $D_{1}, D_{2}, \ldots$, where each $D_{j}$ consists of the primes in an interval $\left(\lambda_{j-1}, \lambda_{j}\right]$, with $\lambda_{j} \approx \lambda_{j-1}^{2}$. More precisely, let $\lambda_{0}=1.9$ and define inductively $\lambda_{j}$ for $j \geqslant 1$ as the largest prime so that

$$
\sum_{\lambda_{j-1}<p \leqslant \lambda_{j}} \frac{1}{p} \leqslant \log 2 .
$$


For example, $\lambda_{1}=2$ and $\lambda_{2}=7$. By Mertens' bounds, we have

$$
\log _{2} \lambda_{j}-\log _{2} \lambda_{j-1}=\log 2+O\left(1 / \log \lambda_{j-1}\right)
$$

and it follows that for some absolute constant $K$,

$$
2^{j-K} \leqslant \log \lambda_{j} \leqslant 2^{j+K} \quad(j \geqslant 0) .
$$

For a vector $\mathbf{b}=\left(b_{1}, \ldots, b_{J}\right)$ of non-negative integers, let $\mathscr{A}(\mathbf{b})$ be the set of square-free integers $a$ composed of exactly $b_{j}$ prime factors from $D_{j}$ for each $j$.

Lemma 4.1. Assume $\mathbf{b}=\left(b_{1}, \ldots, b_{J_{2}}\right)$, with $b_{j}=0$ for $j<J_{1}$. Then

$$
\sum_{a \in \mathscr{A}(\mathbf{b})} \frac{W^{*}(a)}{a} \ll \frac{(\log 4)^{b_{J_{1}}+\cdots+b_{J_{2}}}}{b_{J_{1}} ! \cdots b_{J_{2}} !} \sum_{j=J_{1}}^{J_{2}} 2^{-j+b_{J_{1}}+\cdots+b_{j}} .
$$

Proof. Identical to the proof of Lemma 2.3 in [6], except that we remove the terms corresponding to $d=d^{\prime}$.

We will only consider those intervals $D_{j} \subseteq(w, y]$, that is, only $J_{1} \leqslant j \leqslant J_{2}$, where

$$
J_{1}:=\min \left\{j: \lambda_{j-1}>w\right\}, \quad J_{2}:=\max \left\{j: \lambda_{j} \leqslant y\right\} .
$$

By (4.4), we have

$$
\left|J_{1}-\frac{\log _{2} w}{\log 2}\right| \leqslant K+2, \quad\left|J_{2}-\frac{\log _{2} y}{\log 2}\right| \leqslant K+1 .
$$

With $w_{0}$ sufficiently large, put

$$
M=\frac{\log _{2} w_{0}}{200}, \quad \varepsilon=2^{-200 M-2 K-4}
$$

In the sequel, all statements involving $M$ implicitly assume that $M$ be sufficiently large. By (4.1), (4.5) and (4.6), we have

$$
J_{1} \geqslant 100 M, \quad J_{2}-J_{1} \geqslant 100 M
$$

Let $\mathscr{B}_{k}$ be the set of vectors $\left(b_{J_{1}}, \ldots, b_{J_{2}}\right)$ which satisfy

(a) $b_{J_{1}}+\cdots+b_{J_{2}}=k$;

(b) $\sum_{j=J_{1}}^{J_{2}} 2^{-j+b_{J_{1}}+\cdots+b_{j}} \leqslant 2^{-M}$;

(c) $b_{J_{1}+i-1} \leqslant M+i^{2} \quad(i \geqslant 1)$;

(d) $b_{J_{2}-i+1} \leqslant M+i^{2} \quad(i \geqslant 1)$.

From the definition of $J_{2}$, whenever $\mathbf{b} \in \mathscr{B}_{k}$ and $a \in \mathscr{A}(\mathbf{b})$, we have $a \in \mathscr{P}(w, y)$. By Lemma 4.1, for any $k$ and any $\mathbf{b} \in \mathscr{B}_{k}$ we have

$$
\sum_{a \in \mathscr{A}(\mathbf{b})} \frac{W^{*}(a)}{a} \leqslant \frac{1}{10} \frac{(\log 4)^{k}}{b_{J_{1}} ! \cdots b_{J_{2}} !} .
$$


By (4.4), the fact that $J_{1}$ is sufficiently large, and $b_{j} \leqslant\left(j+1-J_{1}\right)^{2}+M$, for any $k$ and $\mathbf{b} \in \mathscr{B}_{k}$ we have by (4.3)

$$
\begin{aligned}
\sum_{a \in \mathscr{A}(\mathbf{b})} \frac{\tau(a)}{a} & =2^{k} \prod_{j=J_{1}}^{J_{2}} \frac{1}{b_{j} !}\left(\sum_{p_{1} \in D_{j}} \frac{1}{p_{1}} \sum_{\substack{p_{2} \in D_{j} \\
p_{2} \neq p_{1}}} \frac{1}{p_{2}} \cdots \sum_{\substack{p_{b_{j}} \in D_{j} \\
p_{b_{j}} \notin\left\{p_{1}, \ldots, p_{b_{j}-1}\right\}}} \frac{1}{p_{b_{j}}}\right) \\
& \geqslant 2^{k} \prod_{j=J_{1}}^{J_{2}} \frac{1}{b_{j} !}\left(\log 2-\frac{b_{j}}{\lambda_{j-1}}\right)^{b_{j}} \geqslant \frac{(\log 4)^{k}}{2 b_{J_{1}} ! \cdots b_{J_{2}} !} .
\end{aligned}
$$

Combining Lemma 3.1, (4.2), (4.8), and (4.9), we arrive at

$$
H\left(x, y, 2 y ; \mathcal{R}_{w}\right)-H\left(x / 2, y, 2 y ; \mathcal{R}_{w}\right) \gg \frac{x}{\log ^{2} y} \sum_{k_{1} \leqslant k \leqslant k_{2}} \sum_{\mathbf{b} \in \mathscr{B}_{k}} \frac{(\log 4)^{k}}{b_{J_{1}} ! \cdots b_{J_{2}} !}
$$

for any $k_{1} \leqslant k_{2}$. We bound the sum on $\mathbf{b}$ using techniques from [5].

Following our heuristic, take

$$
k_{2}=\left\lfloor\min \left(\frac{\log _{2} y}{\log 2}, 2\left(\log _{2} y-\log _{2} w\right)\right)-2 M\right\rfloor .
$$

By (4.1), $k_{2} \geqslant 100 M$ and by (4.5),

$$
k_{2}=\min \left(J_{2},(\log 4)\left(J_{2}-J_{1}\right)\right)-2 M+\theta, \quad|\theta| \leqslant(\log 4)(2 K+3) .
$$

We will choose $k_{1}$ to satisy

$$
10 M \leqslant k_{1} \leqslant k_{2}
$$

Also define

$$
v=J_{2}-J_{1}+1, \quad s=J_{1}-2-M .
$$

Setting $g_{i}=b_{J_{1}+i-1}$ for $i \geqslant 1$, we have

$$
\sum_{i=1}^{v} 2^{-i+g_{1}+\cdots+g_{i}}=2^{J_{1}-1} f(\mathbf{b}) \leqslant 2^{s+1} .
$$

By (c) and (d) in the definition of $\mathscr{B}_{k}, g_{i} \leqslant M+i^{2}$ and $g_{v+1-i} \leqslant M+i^{2}$ for every $i \geqslant 1$. Applying the argument on the top of page 419 in [5], it follows that for $k_{1} \leqslant k \leqslant k_{2}$ we have

$$
\sum_{\mathbf{b} \in \mathscr{B}_{k}} \frac{(\log 4)^{k}}{b_{J_{1}} ! \cdots b_{J_{2}} !} \gg(v \log 4)^{k} \operatorname{Vol}\left(Y_{k}(s, v)\right)
$$

where $Y_{k}(s, v)$ is the set of $\boldsymbol{\xi}=\left(\xi_{1}, \ldots, \xi_{k}\right) \in \mathbb{R}^{k}$ satisfying

(i) $0 \leqslant \xi_{1} \leqslant \cdots \leqslant \xi_{k}<1$;

(ii) For $1 \leqslant i \leqslant \sqrt{k-M}, \xi_{M+i^{2}}>i / v$ and $\xi_{k+1-\left(M+i^{2}\right)}<1-i / v$;

(iii) $\sum_{j=1}^{k} 2^{j-v \xi_{j}} \leqslant 2^{s}$.

We now invoke a result from [5] concerning the volume of $Y_{k}(s, v)$. 
Lemma 4.2 ([프, Lemma 4.9]). Uniformly for $v \geqslant 1,10 M \leqslant k \leqslant 100(v-1), s \geqslant M / 2+1$ and $0 \leqslant k-v \leqslant s-M / 3-1$. Then

$$
\operatorname{Vol}\left(Y_{k}(s, v)\right) \gg \frac{k-v+1}{(k+1) !} .
$$

Combining (4.6), (4.7), (4.11), (4.12) and (4.13), we find

$$
\begin{aligned}
v & =J_{2}-J_{1}+1 \geqslant 100 M, \\
10 M & \leqslant k_{1} \leqslant k_{2} \leqslant(\log 4)\left(J_{2}-J_{1}\right)=(\log 4)(v-1), \\
s & \geqslant \log _{2} w-M \geqslant M / 2+1, \\
k_{2}-v-s & \leqslant\left(J_{2}-2 M\right)-\left(J_{2}-1-M\right)=1-M \leqslant-M / 3-1 .
\end{aligned}
$$

Thus, the hypotheses of Lemma 4.2 are satisfied for all $k \in\left[k_{1}, k_{2}\right]$. Therefore, gathering (4.10), (4.14) and invoking Lemma 4.2, we conclude that

$$
H\left(x, y, 2 y ; \mathcal{R}_{w}\right)-H\left(x / 2, y, 2 y ; \mathcal{R}_{w}\right) \gg \frac{x}{\log ^{2} y} \sum_{k_{1} \leqslant k \leqslant k_{2}} \frac{(v \log 4)^{k}}{k !}\left(\frac{k-v+1}{k+1}\right) .
$$

Consider three cases: I. $\delta \geqslant 1-\frac{1}{\log 4}$, II. $\frac{1}{5} \leqslant \delta<1-\frac{1}{\log 4}$, III. $0<\delta<\frac{1}{5}$.

Case I. We have $\log _{2} w \geqslant\left(1-\frac{1}{\log 4}\right) \log _{2} y$ and thus, by (4.11) and (4.5),

$$
k_{2}=\left\lfloor 2\left(\log _{2} y-\log _{2} w\right)-2 M\right\rfloor \geqslant v \log 4-2 M-2 K-4 \geqslant 1.1 v .
$$

Now set $k_{1}=0.9 k_{2}$, so that (4.12) is satisfied. Then

$$
\frac{k-v+1}{k+1} \asymp 1 \quad\left(k_{1} \leqslant k \leqslant k_{2}\right) .
$$

Applying Lemma 2.4 to the sum in (4.15), we obtain

$$
H\left(x, y, 2 y ; \mathcal{R}_{w}\right)-H\left(x / 2, y, 2 y ; \mathcal{R}_{w}\right) \gg \frac{x}{\log ^{2} y} e^{v \log 4} \gg \frac{x}{\log ^{2} w},
$$

as required in this case.

Case II. By (4.11), followed by (4.5), we have

$$
k_{2}=\left\lfloor\frac{\log _{2} y}{\log 2}-2 M\right\rfloor \geqslant \frac{5}{4}(v-2 K-4)-2 M \geqslant 1.2 v
$$

and take

$$
k_{1}=\frac{9}{10} k_{2}
$$

Thus,

$$
\frac{k-v+1}{k+1} \asymp 1 \asymp \delta \quad\left(k_{1} \leqslant k \leqslant k_{2}\right) .
$$


We apply Lemma 2.4 with $h=k_{1}, m=k_{2}, x=v \log 4$, and use

$$
\begin{aligned}
\min \left(x^{1 / 2}, \frac{x}{x-m}, m-h+1\right) & \gg \min \left(\left(\log _{2} y\right)^{1 / 2}, \frac{v \log 4}{v \log 4-k_{2}}\right) \\
& \gg \min \left(\left(\log _{2} y\right)^{1 / 2}, \frac{1}{1-\frac{1}{\log 4}-\delta}+O\left(1 / \log _{2} y\right)\right) \\
& \gg \delta\left(\log _{2} y\right)^{1 / 2} B(w, y) .
\end{aligned}
$$

Recalling the definition of $\mathcal{E}$, we have by Stirling's formula,

$$
\frac{(v \log 4)^{k_{2}}}{k_{2} !} \gg \frac{(e(1-\delta))^{k_{2}}}{\sqrt{\log _{2} y}}=\frac{(\log y)^{2-\mathcal{E}+\frac{\log (1-\delta)}{\log 2}}}{\sqrt{\log _{2} y}} .
$$

Invoking Lemma 2.4 we see that the sum in (4.15) is

$$
\gg \delta B(w, y)(\log y)^{2-\mathcal{E}+\frac{\log (1-\delta)}{\log 2}},
$$

and this gives the required lower bound in Theorem 1.

Case III. When $\delta<\frac{1}{10}$, we also have

$$
k_{2}=\left\lfloor\frac{\log _{2} y}{\log 2}-2 M\right\rfloor=J_{2}-2 M+O(K),
$$

but in this case we take

$$
k_{1}=k_{2},
$$

as we are in the range where the summation in (4.15) is dominated by the final summand regardless of $k_{1}$. Here

$$
k_{2}-v+1 \asymp J_{1}, \quad \frac{k_{2}-v+1}{k_{2}+1} \asymp \frac{\log _{2} w}{\log _{2} y}=\delta .
$$

Applying Lemma 2.4 to the sum in (4.15), we obtain

$$
H\left(x, y, 2 y ; \mathcal{R}_{w}\right)-H\left(x / 2, y, 2 y ; \mathcal{R}_{w}\right) \gg \frac{\delta x}{\log ^{2} y} \frac{(v \log 4)^{k_{2}}}{k_{2} !} .
$$

Applying Stirling's formula as in Case II and observing that $B(w, y)=1$ in this case, we conclude the desired upper bound.

This completes the proof of the lower bound in Theorem 1 .

\section{Proof of Theorem 1: UpPer Bounds}

In this section, we prove the upper bound in Theorem 1 We begin with simple cases. If $w_{0}$ is fixed and $w \leqslant w_{0}$, then $H\left(x, y, 2 y ; \mathcal{R}_{w}\right) \leqslant H(x, y, 2 y)$ and the required bound follows from (1.1). Next, if $\log _{2} w \geqslant(1-1 / \log 4) \log _{2} y$, then by Lemma 2.3,

$$
H\left(x, y, 2 y ; \mathcal{R}_{w}\right) \leqslant \sum_{\substack{y<d \leqslant 2 y \\ P^{-}(d)>w}}\left|\left\{m \leqslant x / d: P^{-}(m)>w\right\}\right| \ll \sum_{\substack{y<d \leqslant 2 y \\ P^{-}(d)>w}} \frac{x}{d \log w} \ll \frac{x}{\log ^{2} w},
$$

as required. 
From now on, we assume that

$$
\log w \leqslant(\log y)^{1-1 / \log 4},
$$

that is, $\delta \leqslant 1-\frac{1}{\log 4}$. We apply Lemma 3.1 and use upper bounds for $L(a)$ from Lemma 2.1. As in [5], the sums involving $L(a)$ are bounded in terms of multivariate integrals, which were estimated accurately in [5, 6].

5.1. Case I. $\frac{1}{10} \leqslant \delta \leqslant 1-\frac{1}{\log 4}$. This case is very easy, as we expect no clustering of divisors. Let

$$
k_{0}=\left\lfloor\frac{\log _{2} y}{\log 2}\right\rfloor .
$$

Beginning with Lemma 3.1, we apply Lemma 2.1 (i) to bound $L(a)$ and then apply Lemma 2.2 parts (a) and (b). We have

$$
\begin{aligned}
H\left(x, y, 2 y ; \mathcal{R}_{w}\right) & \ll \frac{x}{\log ^{2} y}\left[\sum_{k \leqslant k_{0}} 2^{k} \sum_{\substack{a \in \mathscr{P}(w, y) \\
\omega(a)=k}} \frac{1}{a}+\sum_{k>k_{0}} \sum_{\substack{a \in \mathscr{P}(w, y) \\
\omega(a)=k}} \frac{\log a}{a}\right] \\
& \ll \frac{x}{\log ^{2} y}\left[\sum_{k \leqslant k_{0}} \frac{\left(2 \log _{2} y-2 \log _{2} w\right)^{k}}{k !}+(\log y) \sum_{k \geqslant k_{0}} \frac{\left(\log _{2} y-\log _{2} w\right)^{k}}{k !}\right] .
\end{aligned}
$$

Since $k_{0} \geqslant 1.4\left(\log _{2} y-\log _{2} w\right)$, the second sum on the right side is dominated by the single term $k=k_{0}$ and thus by Stirling's formula we get that

$$
\sum_{k \geqslant k_{0}} \frac{\left(\log _{2} y-\log _{2} w\right)^{k}}{k !} \ll \frac{\left(\log _{2} y-\log _{2} w\right)^{k_{0}}}{k_{0} !} \ll \frac{((e \log 2)(1-\delta))^{k_{0}}}{\left(\log _{2} y\right)^{1 / 2}} \ll \frac{(\log y)^{1-\mathcal{E}+\frac{\log (1-\delta)}{\log 2}}}{\left(\log _{2} y\right)^{1 / 2}} .
$$

We have $k_{0} \leqslant 2 \log _{2} y-2 \log _{2} w$ in the first sum, for which we invoke Lemma 2.4 and obtain, with $\alpha=\log _{2} y-\log _{2} w$ the bound

$$
\begin{aligned}
\sum_{k \leqslant k_{0}} \frac{\left(2 \log _{2} y-2 \log _{2} w\right)^{k}}{k !} & \ll \frac{(2 \alpha)^{k_{0}}}{k_{0} !} \min \left(\alpha^{1 / 2}, \frac{\alpha}{\alpha-k_{0}}\right) \\
& \ll(2 e(\log 2)(1-\delta))^{k_{0}} \min \left(1,\left(\log _{2} y\right)^{-1 / 2}((1-\delta) \log 4-1)^{-1}\right) \\
& \ll(\log y)^{-\mathcal{E}+\frac{\log (1-\delta)}{\log 2}} B(w, y),
\end{aligned}
$$

as required for Theorem 1

5.2. Case II. $\delta \leqslant \frac{1}{10}$. This case is more delicate, because we expect that typically there will be clustering of the divisors of $a$, and we must bound the probability of non-clustering.

We cut up the sum in Lemma 3.1 according to $\omega(a)$. Let

$$
T_{k}=\sum_{\substack{a \in \mathscr{P}(w, y) \\ \omega(a)=k}} \frac{L(a)}{a} .
$$

We bound $T_{k}$ in terms of a mutivariate integral, in a manner similar to that in [6]. 
Lemma 5.1. Suppose $w$ is large, (5.1) holds, let

$$
v=\left\lfloor\frac{\log _{2} y-\log _{2} w}{\log 2}\right\rfloor, \quad u=\left\lfloor\frac{\log _{2} w}{\log 2}\right\rfloor
$$

and assume that $1 \leqslant k \leqslant 10 v$. Then

$$
T_{k} \ll\left(2 \log _{2} y-2 \log _{2} w\right)^{k} U_{k}(v, u),
$$

where

$$
U_{k}(v, u)=\int_{0 \leqslant \xi_{1} \leqslant \cdots \leqslant \xi_{k} \leqslant 1} \cdots \int_{0 \leqslant j \leqslant k} \min ^{-j}\left(2^{v \xi_{1}+u}+\cdots+2^{v \xi_{j}+u}+1\right) d \boldsymbol{\xi} .
$$

Proof. The proof is the same as the proof of Lemma 3.5 in [6], except that we make use of the fact that $P^{-}(a)>w$. Recall the definition of the sets $D_{j}$ from Section 4 . By (4.4), any prime divisor of $a$ lies in $D_{j}$ with $u-K-2 \leqslant j \leqslant v+u+K+3$. Following the proof of [6, Lemma 3.5], in particular using Lemma 2.1 (iii), we have

$$
T_{k} \ll \frac{(2 \log 2)^{k}}{k !} \int_{[u-K-2, v+u+K+4]^{k}} F(\mathbf{t}) d \mathbf{t},
$$

where, letting $s_{1} \leqslant s_{2} \leqslant \cdots \leqslant s_{k}$ be the increasing rearrangement of $t_{1}, \ldots, t_{k}$,

$$
F(\mathbf{t})=\min _{0 \leqslant j \leqslant k} 2^{-j}\left(2^{s_{1}}+\cdots+2^{s_{j}}+1\right) .
$$

Observe that $F(\mathbf{t})$ is symmetric in $t_{1}, \ldots, t_{k}$. Making the change of variables

$$
t_{i}=u-K-2+(v+2 K+6) \xi_{i} \quad(1 \leqslant i \leqslant k)
$$

we see that $0 \leqslant \xi_{i} \leqslant 1$ for each $i$. Utilizing the summetry of $F(\mathbf{t})$, we find that the multiple integral on the right side equals

$$
(v+2 K+6)^{k} k ! \int_{0 \leqslant \xi_{1} \leqslant \cdots \leqslant \xi_{k} \leqslant 1} \cdots \int_{0 \leqslant j \leqslant k} \min ^{-j}\left(2^{(v+2 K+6) \xi_{i}+u}+\cdots+2^{(v+2 K+6) \xi_{g}+u}+1\right) d \boldsymbol{\xi} .
$$

We conclude that

$$
T_{k}(y) \ll((2 \log 2)(v+2 K+6))^{k} U_{k}(v, u) .
$$

Lastly, $(v+2 K+6)^{k} \ll v^{k}$ since $k \leqslant 10 v$, and the lemma follows.

To bound $U_{k}(u, v)$ we invoke the following estimate from [5, 6].

Lemma 5.2 ([5, Lemma 13.2],[6, Lemma 4.4]). Define

$$
\mathscr{T}(k, v, \gamma)=\left\{\boldsymbol{\xi} \in \mathbb{R}^{k}: 0 \leqslant \xi_{1} \leqslant \cdots \leqslant \xi_{k} \leqslant 1,2^{v \xi_{1}}+\cdots+2^{v \xi_{j}} \geqslant 2^{j-\gamma}(1 \leqslant j \leqslant k)\right\} .
$$

Suppose $k, v, \gamma \in \mathbb{Z}$ with $1 \leqslant k \leqslant 10 v$ and $\gamma \geqslant 0$. Set $b=k-v$. Then

$$
\operatorname{Vol}(\mathscr{T}(k, v, \gamma)) \ll \frac{Y}{2^{2^{b-\gamma}}(k+1) !}, \quad Y=\left\{\begin{array}{ll}
b & \text { if } b \geqslant \gamma+5 \\
(\gamma+5-b)^{2}(\gamma+1) & \text { if } b<\gamma+5
\end{array} .\right.
$$

Lemma 5.3. Suppose $k, u, v$ are integers satisfying $1 \leqslant k \leqslant 10 v$ and $u \geqslant 1$. Then

$$
U_{k}(v, u) \ll \frac{u\left(1+|k-v-u|^{2}\right)}{(k+1) !\left(2^{k-v-u}+1\right)} .
$$

Notice that the bound in Lemma 5.3 undergoes a change of behavior at $k=v+u$. 
Proof. Put $b=k-v$. For integers $m \geqslant 0$, consider $\boldsymbol{\xi}$ satisfying

$$
2^{-m} \leqslant \min _{0 \leqslant j \leqslant k} 2^{-j}\left(2^{v \xi_{1}+u}+\cdots+2^{v \xi_{j}+u}+1\right)<2^{1-m} .
$$

For $1 \leqslant j \leqslant k$ we have

$$
2^{-j}\left(2^{v \xi_{1}+u}+\cdots+2^{v \xi_{j}+u}\right) \geqslant \max \left(2^{-j}, 2^{-m-u}-2^{-j-u}\right) \geqslant 2^{-m-u-1},
$$

and thus $\boldsymbol{\xi} \in \mathscr{T}(k, v, m+u+1)$. Invoking Lemma 5.2, we find that

$$
\begin{aligned}
& U_{k}(v, u) \leqslant \sum_{m \geqslant 0} 2^{1-m} \operatorname{Vol}(\mathscr{T}(k, v, m+u+1)) \ll \frac{1}{(k+1) !} \sum_{m \geqslant 0} \frac{2^{-m} Y_{m}}{2^{2^{b-m-u-1}}}, \\
& Y_{m}=\left\{\begin{array}{ll}
b & \text { if } m+u \leqslant b-6 \\
(m+u+6-b)^{2}(m+u+2) & \text { if } m+u>b-6
\end{array}\right. \text {. }
\end{aligned}
$$

Dividing the sum according to the two cases yields

$$
\sum_{m \geqslant 0} \frac{2^{-m} Y_{m}}{2^{2^{b-u-m-1}}} \ll \sum_{0 \leqslant m<b-u-5} \frac{b}{2^{m} 2^{2^{b-m-u-1}}}+\sum_{m \geqslant \max (0, b-u-5)} \frac{(m+u+6-b)^{2}(m+u+2)}{2^{m}} .
$$

The proof is completed by noting that if $b \geqslant 6+u$, each sum on the right side is $\ll b 2^{u-b}$ and if $b \leqslant 5+u$, the first sum is empty and the second is $\ll(6+u-b)^{2} \ll 1+(b-u)^{2}$.

Finally, we complete the upper bound in Theorem 1. Let $v=\left\lfloor\frac{\log _{2} y-\log _{2} w}{\log 2}\right\rfloor, u=\left\lfloor\frac{\log _{2} w}{\log 2}\right\rfloor$ and define $k_{0}$ by (5.2). Note that $k_{0}=v+u+O(1)$. We now combine Lemmas 5.1 and 5.3 , Since $k_{0}>1.4\left(\log _{2} y-\log _{2} w\right)$, we have

$$
\begin{aligned}
\sum_{k_{0} \leqslant k \leqslant 10 k_{0}} T_{k} & \ll \sum_{k_{0} \leqslant k \leqslant 10 k_{0}} \frac{u\left(1+\left(k-k_{0}\right)^{2}\right)}{(k+1) ! 2^{k-u-v}}\left(2 \log _{2} y-2 \log _{2} w\right)^{k} \\
& \ll u 2^{k_{0}} \sum_{\ell \geqslant 0} \frac{1+\ell^{2}}{\left(k_{0}+1+\ell\right) !}\left(\log _{2} y-\log _{2} w\right)^{k_{0}+\ell} \\
& \ll\left(\log _{2} w\right) \frac{\left(2 \log _{2} y-2 \log _{2} w\right)^{k_{0}}}{\left(k_{0}+1\right) !} .
\end{aligned}
$$

Similarly, since $k_{0} \leqslant 0.9\left(2 \log _{2} y-2 \log _{2} w\right)$, we have

$$
\begin{aligned}
\sum_{0 \leqslant k<k_{0}} T_{k} & \ll 1+\sum_{1 \leqslant k<k_{0}} \frac{u\left(k_{0}-k\right)^{2}\left(2 \log _{2} y-2 \log _{2} w\right)^{k}}{(k+1) !} \\
& \ll 1+u \sum_{\ell=1}^{k_{0}-1} \frac{u \ell^{2}\left(2 \log _{2} y-2 \log _{2} w\right)^{k_{0}-\ell}}{\left(k_{0}+1-\ell\right) !} \\
& \ll\left(\log _{2} w\right) \frac{\left(2 \log _{2} y-2 \log _{2} w\right)^{k_{0}}}{\left(k_{0}+1\right) !} .
\end{aligned}
$$


For the large values of $k$ we use the crude bound $L(a) \ll \tau(a)$ from Lemma 2.1 (i), followed by an application of Lemma 2.2 (a). This gives

$$
\begin{aligned}
\sum_{k \geqslant 10 k_{0}} T_{k} & \leqslant \sum_{k \geqslant 10 k_{0}} \sum_{\substack{a \in \mathscr{P}(w, y) \\
\omega(a)=k}} \frac{2^{k} \log 2}{a} \leqslant \sum_{k \geqslant 10 k_{0}} \frac{\left(2 \log _{2} y-2 \log _{2} w+O(1)\right)^{k}}{k !} \\
& \ll \frac{\left(2 \log _{2} y-2 \log _{2} w+O(1)\right)^{10 k_{0}}}{\left(10 k_{0}\right) !} \\
& \ll \frac{\left(2 \log _{2} y-2 \log _{2} w\right)^{k_{0}}}{\left(k_{0}+1\right) !} .
\end{aligned}
$$

Combining these three bounds for sums of $T_{k}$ with Lemma 3.1, Lemma 2.4, and Stirling's formula, we conclude that

$$
\begin{aligned}
H\left(x, y, 2 y ; \mathcal{R}_{w}\right) & \ll \frac{x}{\log ^{2} y}\left(\log _{2} w\right) \frac{\left(2 \log _{2} y-2 \log _{2} w\right)^{k_{0}}}{\left(k_{0}+1\right) !} \\
& \ll \frac{x \log _{2} w}{\left(\log _{2} y\right)^{3 / 2}}(\log y)^{-\mathcal{E}+\frac{\log (1-\delta)}{\log 2}} .
\end{aligned}
$$

The proof of the upper bound in Theorem 1 is complete.

\section{REFERENCES}

1. P. Erdős and A. Schinzel, On the greatest prime factor of $\prod_{k=1}^{x} f(k)$, Acta Arith. 55 (1990), no. 2, 191-200. MR 1061638

2. P. Erdős, On the greatest prime factor of $\prod_{k=1}^{x} f(k)$, J. London Math. Soc. 27 (1952), 379-384. MR 0047686

3. __ Some remarks on number theory, Riveon Lematematika 9 (1955), 45-48, (Hebrew. English summary). MR 17,460d

4. _ An asymptotic inequality in the theory of numbers, Vestnik Leningrad. Univ. 15 (1960), no. 13, 41-49, (Russian). MR 23 \#A3720

5. K. Ford, The distribution of integers with a divisor in a given interval, Ann. Math. (2008), 367-433.

6. _ Integers with a divisor in $(y, 2 y]$, Anatomy of Integers, CRM Proceedings and Lecture Notes (2008), 65-80, Proof simplified in arXiv: math/0607473.

7. K. Ford, M. R. Khan, I. E. Shparlinski, and C. L. Yankov, On the maximal difference between an element and its inverse in residue rings, Proc. Amer. Math. Soc. 133 (2005), no. 12, 3463-3468. MR 2163580

8. H. Halberstam and H.-E. Richert, Sieve methods, Academic Press, 1974, London Mathematical Society Monographs, No. 4. MR 54 \#12689

9. D. Koukoulopoulos, Divisors of shifted primes, Int. Math. Res. Not. IMRN 2010 (2010), no. 24, 45854627.

10. _ Localized factorizations of integers, Proc. London Math. Soc. 101 (2010), 392-426.

11. K. K. Norton, On the number of restricted prime factors of an integer. I, Illinois J. Math. 20 (1976), no. 4, 681-705. MR 54 \#7403

12. G. Tenenbaum, Sur une question d'Erdős et Schinzel, A tribute to Paul Erdős, Cambridge Univ. Press, Cambridge, 1990, pp. 405-443. MR 1117034

13. __ Sur une question d'Erdős et Schinzel. II, Invent. Math. 99 (1990), no. 1, 215-224. MR 1029397

Department of Mathematics, 1409 West Green Street, University of Illinois at UrbanaChampaign, Urbana, IL 61801, USA

E-mail address: ford126@illinois.edu 